\title{
V. MILZ UND PANKREAS
}

\section{V.1 Zuckergussmilz}

\section{Kasuistik}

Eine 76-jährige Rentnerin, die 1961 wegen einer Schenkelhalsfraktur zur Aufnahme gelangt und 7 Tage danach wegen einer Lobärpneumonie und kardialer Insuffizienz verstirbt. Bei der Obduktion wird zusätzlich eine Fettembolie als Todesursache festgestellt.

\section{Morphologische Untersuchung}

Eine 15:10:8 cm messende Milz mit einer zuckergussartigen glatten, festen, gelblich-weißlichen Kapsel. Diese auf der Schnittfläche bis $5 \mathrm{~mm}$ dick und schwartenartig. Das Parenchym verfestigt, rote und weiße Pulpa gut erkennbar. Histologisch die Kapsel aus einem hyalinisierten Bindegewebe bestehend. Subkapsulär reichlich Hämosiderophagen.

\section{Diagnose}

Perisplenitis pseudocartilaginea - Zuckergussmilz MN 23.733 (Abb. V.1 $\rightarrow$ Farbbild S. 180).

\section{Definition}

Eine durch eine Entzündung oder Stauung ausgelöste schwartige, hyaline ${ }^{67}$ Verdickung der Milzkapsel.

\section{Ätiologie/Pathogenese}

Als Hyalin bezeichnet man histologisch eine vorwiegend aus Proteinen bestehende glasig-homogene Ablagerung, welche sich intra- und extrazellulär darstellen kann und sich mit Eosin rot anfärben lässt. Im Fall der Zuckergussmilz handelt es sich um extrazellulär abgelagertes bindegewebiges Hyalin. Eine plattenartige Verdickung der serösen Häute im Sinne einer hyalinen knorpelartigen Wandverdickung weist auf eine abgelaufene chronische Entzündung (z.B. abgeheilte Perisplenitis) hin. Weitere Ursachen sind u.a. ein Aszites oder ein chronisches Kapselödem.

\section{Morphologie}

Durch das Hyalin erscheint die - entzündlich bedingt knorpelartig verdickte Kapsel der vergrößerten Milz weißlich und erinnert an einen Zuckerguss, der der Zuckergussmilz auch ihren Namen

67 griech.: hyalos = gläsern, durchsichtig. 
verdankt. Von der grauvioletten Schnittfläche lässt sich nur wenig Pulpabrei abstreifen, da auch das Milzparenchym verfestigt ist. Dies im Gegensatz zur akuten entzündlichen Milzschwellung bei der sich durch das proteolytisch aufgelockerte Parenchym reichlich Pulpa abstreifen lässt.

Histologisch betrachtet, besteht dieses Hyalin aus einem dichten und zellarmen Kollagenfaserfilz.

\section{V.2 M. Gaucher}

\section{Kasuistik}

Ein 7 Monate altes Mädchen, das mit 3 Monaten wegen ständigen Mundhöhlenrasseln und partieller Schlucklähmung aufgenommen wurde. In einem entnommenen Knochenmarkspunktat fanden sich große, wabige Zellen, die als fragliche Gaucher-Zellen interpretiert wurden. Zwei Monate nach Aufnahme traten eine progredient zunehmende Hepatosplenomegalie und weitere Schluckstörungen auf, die ein ständiges Absaugen notwendig machten. Exitus unter den Zeichen der bulbären Paralyse.

\section{Morphologische Untersuchung}

Deutlich vergrößerte Leber mit abgerundeten Rändern und eine ebenso vergrößerte, wurstartige Milz. Histologisch die Milzpulpa blutarm mit umfänglichen Zellansammlungen, die die Sinusoide zur Gänze erfüllen. Die großleibigen Zytoplasmen zart eosinophil, teils knittrig imponierend. In der Leber ebenso reichlich homologe Zellansammlungen.

\section{Diagnose}

M. Gaucher ${ }^{68}$ (Abb. V.2 $\rightarrow$ CD-ROM).

\section{Definition}

Eine autosomal rezessive Lipidspeicherkrankheit, die durch eine pathologische Speicherung von Zerebrosiden im retikuloendothelialen System und ggf. im ZNS gekennzeichnet ist.

68 Philippe Ch. E. Gaucher (1854-1918), Hautarzt, Paris. 
Synonyme: Glukozerebrosidose, Glucosylceramidlipidose.

\section{Geschichtliche Notizen}

1882 beschrieb der französische Medizinstudent Phillipe Charles Ernest Gaucher den Fall einer 32-jährigen Frau mit einer ungewöhnlich ausgeprägten Splenomegalie. In seiner Doktorarbeit fasste er klinische Symptome und morphologische Veränderungen zusammen. In der Histologie des Autopsiepräparates fand er auffallend vergrößerte Zellen, die wie sich später herausstellte, pathognomonisch für diese Erkrankung sind. Ihm zur Ehre tragen sie heute seinen Namen - Gaucher-Zellen.

\section{Ätiologie/Pathogenese}

Bei der autosomal rezessiv vererbten Glucosylceramidlipidose weist die lysosomale $\beta$-Glukosidase in den Phagozyten ( = Cerebrosidhydrolase) eine reduzierte Aktivität auf und definiert damit die adulte und häufigere Form: chronischer, nicht neuropathischer M. Gaucher. Eine nicht nachweisbare Aktivität findet sich bei der selteneren, juvenilen Form, die als akuter M. Gaucher eine Mitbeteiligung der Ganglienzellen aufweist und zu Spasmen führt. Der Mangel an der sauren lysosomalen $\beta$-Glukosidase führt zu einem unvollständigen Abbau der lipidhaltigen Zellmembranen in den Zellen des redikuloendothelialen Systems (vor allem von den Erythrozytenmembranen) mit der Bildung zerebrosidhältiger Vakuolen ( $\rightarrow$ Gaucher-Zellen). Ähnliche Vakuolen findet man dann bei der neuropathischen Form auch in Nervenzellen und Gefäßendothelien. Betroffen sind meist Frauen jüdischer Abstammung. Der Gendefekt ist am Chromosom 1q21 beheimatet.

\section{Klinik}

Gesteigerte Proliferation und expansives Wachstum dieser Zellen führen zu einer Vielzahl von Symptomen wie Hepatosplenomegalie und Lymphadenopathie. Man unterscheidet 3 Typen des Morbus Gaucher: (1) adulte, (2) infantile und (3) juvenile Form. Die infantile und schwerste Form ist eine akut neuropathische mit Auftreten von Krämpfen, Spastizität und Lähmungen in den ersten 3 Lebensmonaten, sowie raschem Verlauf und Tod in den ersten 2 Lebensjahren. Die juvenile, subakute Form manifestiert sich bei Kindern und Jugendlichen. Die häufigste, adulte Form geht mit einer Hepatosplenomegalie einher. Folgen sind Infektionen des Respirationstrakts, neurologische Ausfälle, Knochenschmerzen und thrompozytopenische Blutungen.

\section{Morphologie}

Das besondere pathologische Kennzeichen des Morbus Gaucher ist die Gaucher-Zelle (20-200 $\mu \mathrm{m}$ im Durchmesser mit homogenem, PAS-positiven Zytoplasma; elektronenmikroskopisch: unregelmäßige Bündel oft verdrillter tubulärer Strukturen, im Längsschnitt fischzug-ähnlich, im Querschnitt als Ringe erscheinend). Es handelt sich um eine ballonierte rundliche Retikulumzelle mit einem kleinen exzentrischen Kern und einem schaumigen, seidenpapierartig zerknitterten Zytoplasma. Morphologisch besteht eine erhebliche Hepatosplenomegalie, eine Lymphknotenvergrößerung und eine Osteoporose bei der adulten Form infolge von Knochenmarksherden. Im Knochenmark sind die Gaucher-Zellen entweder diffus verteilt oder bilden tumorartige Bezirke, die den Knochen auftreiben, arrodie- 
ren und damit auch zu pathologischen Frakturen prädisponieren. Die distalen Femura können typischer Weise Erlenmeyerkolben-förmig aufgetrieben sein.

\section{Literatur}

Aerts JM, Hollak CE, van Breemen M, Maas M, Groener JE, Boot RG (2005) Identification and use of biomarkers in Gaucher disease and other lysosomal storage diseases. Acta Paediatr Suppl 94 (447): 43-6; discussion 37-38.

Grabowski GA (2005) Recent clinical progress in Gaucher disease. Curr Opin Pediatr 17 (4): 519-24.

Jmoudiak M, Futerman AH (2005) Gaucher disease: pathological mechanisms and modern management. Br J Haematol 129 (2): $178-88$.

\section{V.3 Schinkenmilz}

\section{Kasuistik}

Eine 50-jährige Frau. Keine weiteren Angaben.

\section{Morphologische Untersuchung}

Eine vergrößerte 15:10:6cm messende, derbe und brüchige Milz mit wachsartiger Schnittfläche. Histologisch interfollikuläre hyaline Massen, die in der Kongorotfärbung unter polarisiertem Licht apfelgrün aufleuchten.

\section{Diagnose}

Schinkenmilz MN 15.837 - Amyloidose der Milz (Abb. V.3 $\rightarrow$ Farbbild S. 181).

\section{Definition}

Bei der Schinkenmilz handelt es sich um eine diffuse Veränderung der Milz im Rahmen von Amyloidablagerungen im Pulparetikulum, in den Nierenglomerula und den Organarterien. Folge kann eine Splenomegalie (Milzgewicht > $500 \mathrm{~g}$ ) mit einem Hyposplenismus sein.

\section{Geschichtliche Notizen}

Rudolf Virchow definierte den Begriff 1854 erstmals und leitete die Bezeichnung aus dem griechischen Wort für Kartoffelstärke ab, da das bei der Sektion gewonnene Material stärkeähnliche Eigenschaften bei der Färbung aufwies.

\section{Ätiologie/Pathogenese}

Bei der Amyloidose handelt es sich um eine Stoffwechselerkrankung bei der es zu einer diffusen Ablagerung von $\beta$ - 
Fibrillen im Extrazellularraum kommt. Amyloid ist ein chemisch nicht einheitlicher Protein-Kohlenhydratkomplex mit charakteristischen Färbeeigenschaften und kommt im Organismus unter normalen Bedingungen nicht vor. Die Amyloidproduktion beruht auf einer fehlerhaften Proteinsynthese. Die Grundbausteine werden zwar intrazellulär gebildet, jedoch per definitionem extrazellulär als Amyloid abgelagert. Es gibt eine Vielzahl verschiedener Amyloidarten, wie z. B. klassisches Amyloid, Immunamyloid, endokrines-, familiäres-, Alters-, Hämodialyse- und Hautamyloid. Alle sind aufgebaut aus einer Mikrofibrillenstruktur um eine röhrenförmige Amyloid-P-Komponente (ein Glykoproteinkernstück), wobei jeweils zwei Proteinfibrillen spiralig um den Kern gedreht und in sich ziehharmonikaartig gefaltet sind. Diese $\beta$-Faltblattstruktur ist charakteristisch für das Amyloid und für die besonderen Färbeeigenschaften verantwortlich.

Prinzipiell unterscheidet man eine primäre Amyloidose (unbekannter Ursache) von einer sekundären. Häufiger ist die sekundäre Form, die so genannte Begleitamyloidose, die als Folge verschiedener chronischer Grunderkrankungen nach monate- oder jahrelangem Verlauf auftritt, bei welchen sich ein mehr oder weniger stark ausgeprägter Eiweißzerfall findet. Hierzu zählen chronisch infektiöse Erkrankungen wie die Tbc, Lues, Lepra. Auch im Gefolge chronischer nicht-infektiöser Krankheiten wie z.B. der primär chronischen Polyarthritis, der Sarkoidose, der Spondyloarthritis ankylopoetica (M. Bechterew), der Colitis ulcerosa, Kollagenosen und dem Morbus Crohn kann sich eine Amyloidose entwickeln. Klassisch ist das Auftreten auch bei Malignomen wie dem M. Hodgkin- bzw. Non-Hodgkin-Lymphom und anderen maligne Erkrankungen.

Im Rahmen dieser sekundären Amyloidose ist zu 100 \% die Milz befallen, ebenso kommt es aber auch zum Befall von Leber, Nebennieren, Nieren, Mukosa und Submukosa des Magen-Darm-Traktes sowie der Lymphknoten.

\section{Klinik}

Milz: Aufgrund der Amyloidose der Milz entwickelt sich u.U. ein Hyposplenismus, der durch eine erhöhte Infektanfälligkeit gegen virale und bakterielle Infektionen mit Begünstigung der Ausbildung einer Sepsis auftreten kann.

Niere: Bei Befall der Niere zeigt sich anfangs eine Polyurie mit geringer $\mathrm{Al}$ buminurie, wobei sich aber im weiteren Verlauf ein nephrotisches Syndrom mit Ausbildung eines Nierenversagens entwickeln kann.

Magen/Darmkanal: Die Mitbeteiligung des Gastrointestinaltraktes mit Ablagerungen in der Mukosa und Submukosa bewirken eine Malabsorption, Diarrhoen und Blutungen.

Nebenniere: Entwicklung eines Morbus Addison.

Herz/Gefäße: Außerdem kann es zu Störungen der Herzfunktion mit Leistungsverminderung (Myokardatrophie) und Rhythmusstörungen (bei Erfassen des Reizleitungssystems), sowie zum Auftreten einer kongophilen Angiopathie, z. B. der Herzkranzgefäße kommen.

Gehirn/Nerven: kongophile Angiopathie der Cerebralarterien, Alzheimer-Krankheit, Sensibilitätsstörungen.

Varia: Ebenso wurden Verdickungen der Gelenkskapseln und das Entstehen einer Makroglossie beschrieben. 


\section{Formen}

\section{$\mathrm{AA}^{69}$-Amyloidose}

Das Serum-Amyloid-A ist ein so genanntes Akute-Phase-Protein, wobei dessen vermehrte Produktion im Rahmen des autosomal-rezessiv vererbten Mittelmeerfiebers auftritt. Aber auch chronische Infektionen, wie z.B. die Tuberkulose, Lepra oder eine Osteomyelitis sind Auslöser. Unter den nichtinfektiösen Ursachen finden sich der Morbus Bechterew, verschiedene Kollagenosen, die Colitis ulcerosa, der Morbus Crohn, die Psoriasis vulgaris, die rheumatoide Arthritis, sowie Malignome. Die AAAmyloidose tritt meist generalisiert auf und betrifft besonders Leber, Niere, Milz und Nebennieren.

\section{AL-Amyloidose}

Diese Form ist gekennzeichnet durch die Vermehrung von Leichtketten der Immunglobuline oder deren Fragmenten. Diese lassen sich als so genannte BenceJones-Eiweise im Harn nachweisen. Meist ist ein Lymphom der Auslöser, wobei neben dem Plasmozytom (multiples Myelom) auch der Morbus Waldenström typische Grunderkrankungen sind. Die AL-Amyloidose entspricht der früheren „primären Amyloidose“. Sie tritt meist lokalisiert in einzelnen Organen wie dem Herzen, der Lunge auf, wobei aber auch bevorzugt Gelenke, Muskeln und Sehnenscheiden miterfasst werden.

\section{AE-Amyloidose}

Die endokrine Form entwickelt sich aus hormonproduzierenden Tumoren, des Pankreas oder der Schilddrüse. Betroffen sind Drüsen, Herz, Niere, Lunge, Nerven und auch die Haut.

69 Die Buchstabenkombination setzt sich aus A für Amyloidose und der betroffenen Proteingruppe zusammen.

\section{AB-Amyloidose}

Hier ist das $\beta$-2-Mikroglobulin für die Genese verantwortlich. Dieses Protein entsteht nach langjähriger Dialyse. Typischerweise kommt es zu schmerzhaften Gelenkskapselablagerungen und auch zu einem Karpaltunnelsyndrom.

\section{AP-Amyloidose}

Die Vorstufe des Albumins, das Präalbumin ist verantwortlich für Ablagerugen in Nerven und Herz.

\section{AS-Amyloidose}

Bei der senilen Amyloidose sind die auslösenden Proteine noch nicht alle bekannt. Eine Häufung ist beim M. Alzheimer festgestellt worden.

\section{Morphologie}

Auffällig ist eine Konsistenzzunahme und ein opakes Parenchym. Die Milz erscheint durch die Amyloidablagerung vergrößert, hart und brüchig, auf der Schnittfläche glasig-transparent und wachsartig matt sowie durch die Kapillarkompression ischämisch blass. Je nach Blutgehalt unterscheidet man eine blassgraue Speckmilz von einer rötlichgrauen Schinkenmilz (vergleichbar mit gekochtem Schinken - s. Abb. V.3B $\rightarrow$ Farbbild S. 181). Histologisch sieht man extrazelluläre Ablagerungen von Amyloid in der roten Pulpa mit Aussparung der Malphigischen Körperchen. Bei isoliertem Befall der weißen Pulpa spricht man hingegen von einer Sagomilz, da die hervorspringenden Malphigischen Körperchen den Eindruck von Hirsekörner machen.

Die $\beta$-Fibrillenstruktur der Amyloidoseplaques färbt sich im Kongorot an und zeigt im polarisierten Licht eine Doppelbrechung mit apfelgrünem Aufleuchten. Der makroskopische Nachweis gelingt durch die Lugol-Probe. 


\section{V.4 M. Hodgkin}

\section{Kasuistik}

Keine Angaben.

\section{Morphologische Untersuchung}

Eine deutlich vergrößerte, wurstartige Milz mit knotig fasrigem Parenchym von derber Konsistenz und mehrfach eingerissener und abgelöster Kapsel. Histologisch ein knotig umstrukturiertes Milzgewebe, wobei die Knoten von sklerotischem Bindegewebe umgeben sind. Das neoplastische, lymphatische Gewebe enthält immer wieder Hodgkin-Zellen sowie Paltauf-Sternberg-Reed Riesenzellen teilweise mit Ausbildung optisch leerer Lakunen.

\section{Diagnose}

M. Hodgkin ${ }^{70}$ MN 24.473 - nodulär sklerosierender Typ (Abb. V.4).

\section{Definition}

Das Hodgkin-Lymphom ist ein monoklonales Malignom des lymphatischen Systems, das durch mononukleäre Hodgkin-Zellen und doppel(mehr-)kernige Sternberg-Reed-(Paltauf)-Zellen sowie einem Mischinfiltrat nicht neoplastischer Leukozyten und Fibroblasten charakterisiert ist.

Synonyme: M. Hodgkin, obsolet: Lymphogranulomatose

Englisch: Hodgkin's disease

70 Thomas Hodgkin, Pathologe in London, geb. am 17. August 1798 in Pentonville, St. James Parish, Middlesex; gest. am 5. April 1866 in Jaffa, Palästina - heute Tel Aviv-Yafo, Israel.

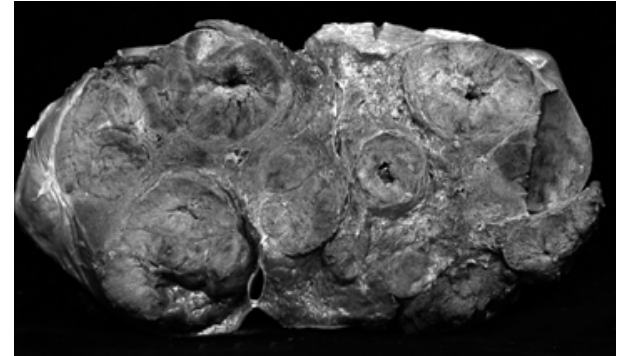

Abb. V.4. MN 24.473. Morbus Hodgkin

\section{Geschichtliche Notizen}

Dieses Malignom wurde erstmals von Thomas Hodgkin 1832 beschrieben und von Samuel Wilks unabhängig von diesem genauer skizziert, wobei Wilks aber in Anerkennung der früheren Publikation Hodgkins das Krankheitsbild nach diesem benannte. Richard Paltauf (18581924) gibt 1896 eine erste Beschreibung der mehrkernigen Riesenzellen, die aber erst 1897 abgedruckt wurde. Carl Sternberg (1872-1935), sein Schüler, erwähnt die Riesenzellen erneut in seiner eigenen Arbeit, die 1898 erschienen ist. Die Wiener Pathologen Paltauf und Sternberg selektierten aus einer großen Zahl von Lymphdrüsentumoren die Entitäten der Kundratschen Lymphosarkomatose, der Myelome und der Pseudolymphome, wobei aus letzterer die Gruppe der Lymphogranulomatose herausgenommen wurde. Beide hielten diese für eine Sonderform der Tuberkulose. Dorothy Reed (1874-1964), eine amerikanische Pathologin, sah diese Assoziation als kritisch und (wieder-)entdeckte 1902 die PaltaufSternberg-Riesenzellen.

\section{Ätiologie/Pathogenese}

Unter den Ursachen wird schon lange die Mitbeteiligung des Ebstein-BarrVirus (EBV) vermutet, doch sind genug 
EBV negative Hodgkin-Fälle bekannt. In den EBV positiven Geweben konnte das Virus in den Sternberg-Reed-Riesenzellen entdeckt werden. Unklar ist ebenso die Genese der Hodgkin- und Sternberg-Reed-Riesenzellen. In $98 \%$ der klassischen Hodgkin-Fälle lässt sich eine B-Zellen-Abstammung nachweisen. Zytogenetisch wurden bisher keine wiederkehrenden oder spezifischen Chromosomenanomalien festgestellt. Die komparative genomische Hybridisierung zeigte allerdings Zugewinne bzw. Überamplifikationen im Bereich der Chromosomen 2p, 9p und 12q. Für aktuelle Forschungsergbnisse siehe Literatur (Campbell, 2005; Re, 2005).

\section{Klinik}

Unter den Lymphomen ist das HodgkinLymphom $\mathrm{zu} 30 \%$ vertreten und wird heute nach WHO in eine klassische und eine noduläre, lymphozyten-prädominante Form aufgrund unterschiedlicher Klinik und Histologie unterteilt. Der klassische Hodgkin weist vier Subtypen nach Rye auf: nodulär-sklerosierend, gemischt, Lymphozyten-arm, Lymphozyten-reich. Epidemiologisch findet sich eine Häufung des klassischen Typs zwischen dem 15. und 35. und als zweiter Gipfel zwischen dem 55. und 65. Lebensalter. Auch familiäre und geografische Häufungen sind beschrieben worden. Patienten mit einer infektiösen Mononukleose (Pfeiffersches Drüsenfieber) in ihrer Anamnese sind häufiger betroffen. Typisch ist eine schmerzlose, zervikale Lymphknotenschwellung, aber auch eine Lymphadenopathie mehrerer peripherer Lymphknotenstationen und des Mediastinums. Zu den so genannten B-Symptomen zählen ein ungewollter Gewichtsverlust von mehr als $10 \%$ des
Körpergewichts innerhalb der letzten 6 Monate, undulierendes Fieber mit Temperaturen über $38{ }^{\circ} \mathrm{C}$ (Pel-EbsteinFieber) und Nachtschweiß. Unspezifisch stellt sich auch Hautjucken und ein Alkoholschmerz in betroffenen Lymphknoten ein.

Anfangs fühlen sich die tastbaren Lymphknoten (LKN) gummiartig an und sind gut verschieblich. Mit Fortschreiten der Erkrankung entwickeln sich miteinander verbackene Lymphknotenpakete. Mediastinal können die vergrößerten LKN den venösen Blutfluss behindern, wodurch eine obere Einflussstauung mit Halsschwellung entsteht (Stokes-Kragen). Die Milz ist häufig mitbeteiligt, sodass sich eine Splenomegalie entwickelt.

Je nach Befall unterscheidet man 4 Stadien nach Ann-Arbor:

Stadium 1: Befall einer Lymphknotenregion oder einer extralymphatischen Lokalisation

Stadium 2: Zwei benachbarte Lymphknotengruppen auf der gleichen Seite des Zwerchfells und einer extralymphatischen Lokalisation

Stadium 3: Beide Seiten des Zwerchfells betroffen

Stadium 4: disseminierter Organbefall mit/ohne LKN-Beteiligung

Für die Prognose ist diese Ann-ArborKlassifikation sowie die klinische BSymptomatik entscheidend.

\section{Morphologie}

Makroskopisch sind die LKN vergrößert und weisen auf der Schnittfläche ein fischfleischartiges Gewebe mit teils knotigem Aufbau, teils regressiven Veränderungen in Form von Nekrosen auf.

Die Milz zeigt weißliche $2 \mathrm{~mm}$ bis $2 \mathrm{~cm}$ großen Knoten, wodurch der Eindruck einer grobgeriebenen Wurst ( = Bauern- 
wurstmilz, Porphyrmilz) entsteht. Voll entwickelt ist die Milz von großem knotigen Tumorgewebe mit fibrösen Bändern durchsetzt.

Für die histologische Diagnose sind die Hodgkin- und die Sternberg-Reed-Riesenzellen typisch. Hodgkin-Zellen sind große Blasten mit schmutzig braunen, auffällig großen Nukleolen ( = Eulenaugenzellen). Sternberg-Reed-Riesenzellen entstehen durch Fusion zweier oder mehrerer Hodgkin-Zellen und sind dann 15-45 $\mu \mathrm{m}$ groß. Die Zellkerne sind oft spiegelbildlich angeordnet und werden von einem breiten graubläulichen Zytoplasma umgeben. Immer wieder werden, bedingt durch die Fixierung, Schrumpfungsartefakte als optisch leere, die Zellen umgebende Höfe gesehen, ein Phänomen, das zum Namen der Lakunarzellen führte. Das begleitende, nichtneoplastische Infiltrat besteht aus Lymphozyten, Plasmazellen, neutrophilen und eosinophilen Granulozyten, Histiozyten und Fibroblasten.

Die klassische Form ist mit der Expression von CD30 und CD15 charakterisiert, die B-Zell-Abstammung wird durch die häufige Positivität vom B-Zell-spezifischen Aktivatorprotein (BSAP) untermauert. Darüber hinaus sind eine Reihe von spezifischen Zytokinen und Chemokinen immunhistochemisch nachweisbar. Ebenso lässt sich das EBV-codierte LMP1 detektieren.

\section{Literatur}

Campbell LJ (2005) Cytogenetics of lymphomas. Pathology 37 (6): 493-507.

Hodgkin T (1832) On some morbid appearances of the absorbent glands and spleen. London: Longman, Rees, Orme, Brown, Green and Longman. Medico-Chirurgical Transactions 17: 68-114
Keegan TH, Glaser SL, Clarke CA, Gulley ML, Craig FE, Digiuseppe JA, Dorfman RF, Mann RB, Ambinder RF (2005) EpsteinBarr virus as a marker of survival after Hodgkin's lymphoma: a population-based study. J Clin Oncol 23 (30): 7604-13.

Paltauf R (1897) Progressive Störungen. a) Neubildungen. b) Lymphosarkom (Lymphosarkomatose, Pseudoleukämie, Myelom, Chlorom). Erg Allg Path Path Anat 3: 652-691.

Re D, Kuppers R, Diehl V (2005) Molecular pathogenesis of Hodgkin's lymphoma. J Clin Oncol 23 (26): 6379-86.

Reed D (1902) On the pathological changes in Hodgkin's disease, with especial reference to its relation to tuberculosis. Johns Hopkins Hospital Reports 10: 133-196.

Stein H, Delsol G, Pileri S et al (2001) Classical Hodgkin lymphoma. In: Pathology and genetics of tumours of haematopoietic and lymphoid tissues (Jaffe ES, Harris NL, Stein H, Vardiman JW, eds), WHO Classifications of tumours. Lyon: IARCPress.

Sternberg C (1898) Über eine eigenartige, unter dem Bilde der Pseudoleukämie verlaufende Tuberkulose des lymphatischen Apparates. Zs Heilk Prag 19: 21-90.

Wilks S (1856) Enlargements of the lymphatic glands and spleen (or Hodgkin's disease) with remarks. Guy’s Hosp Rep 11: 56. 


\section{V.5 Lymphangioma cavernosum cysticum - Cystadenoma pancreatis}

\section{Kasuistik}

77-jährige Frau, die 1956 an der I. Chirurgischen Klinik verstarb.

\section{Morphologische Untersuchung}

Ein 4,5:5:5 cm messender multizystischer Knoten mit glatten, wabenartigen und dünnwandigen Zysten. Außen anhaftend druckatrophes Pankreasparenchym. Histologisch werden die Zysten von einem PAS positiven, D-PAS negativen isoprismatischen, hellzelligen Epithel ohne Atypien ausgekleidet. Die Zystenwände aus unterschiedlich dickem Bindegewebe bestehend.

\section{Diagnose}

Ursprünglich: MN 6972 Lymphangioma cavernosum cysticum (Abb. V.5).

Heute: Seröses, mikrozystisches Adenom des Pankreas.

\section{Definition}

Das seröse Zystadenom des Pankreas ist ein benigner, multizystischer, epithelialer Tumor, der von einem serösem Epithel ausgekleidet wird.

\section{Ätiologie/Pathogenese}

Diese gutartige Veränderung des Pankreas ist selten und wird zumeist bei Frauen im höheren Lebensalter als $\mathrm{Zu}$ fallsbefund entdeckt.

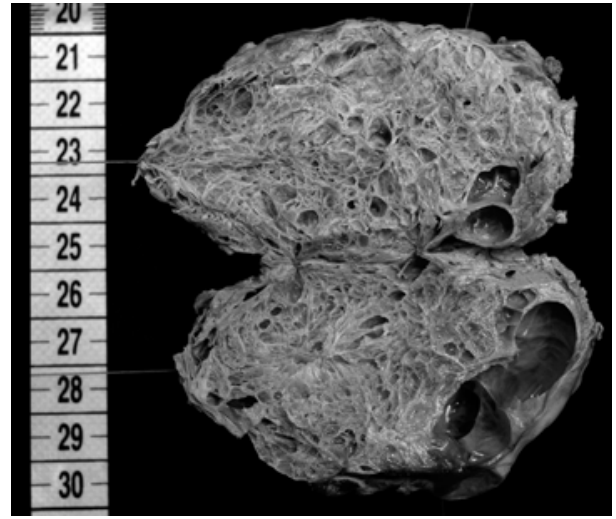

Abb. V.5. MN 6972. Seröses, mikrozystisches Adenom des Pankreas

Klinik

Das seröse mikrozystische Adenom macht wenige bis gar keine Beschwerden. Befindet sich der Tumor im Bereich des Pankreaskopfes, so kann dieser eine Obstruktionspankreatitis oder einen Verschlussikterus hervorrufen. Eine maligne Entartung ist nicht bekannt.

\section{Morphologie}

Das Adenom hat bei Diagnosestellung durchschnittlich eine Größe zwischen 6-10 cm. Es besteht aus kleinen Zysten mit serösem Inhalt, die meist um eine zentrale sternförmige Narbe angeordnet sind. Die Schnittfläche weist eine typische schwammartige Struktur auf. Das histologische Bild ist geprägt von kleinen Zysten, welche von einem isoprismatischen Epithel ausgekleidet sind. Aufgrund des Glykogenreichtums ist das Zytoplasma klarzellig.

\section{Literatur}

Klöppel G, Kosmahl M (2001) Cystic lesions and neoplasms of the pancreas. The features are becoming clearer. Pancreatology 1 (6): 648-55. 


\section{V.6 Pigmentatio melanotica lienalis (Malaria Milz)}

\section{Kasuistik}

Keine Angaben.

\section{Morphologische Untersuchung}

Eine deutlich vergrößerte, braunschwärzliche, brüchige Milz mit derber Konsistenz. Histologisch reichlich Siderophagen und schwärzliche Pigmenteinlagerungen sowie vereinzelt in den Blutgefäßen kleine Haufen von Merozoiten.

\section{Diagnose}

Pigmentatio melanoticalienalis (Malaria ${ }^{71}$ Milz) MN 6922 (Abb. V.6 $\rightarrow$ CD-ROM).

\section{Definition}

Malaria ist eine in den Tropen und Subtropen häufige Zoonose in dessen Rahmen sich ein Milztumor mit bis zu $1000 \mathrm{~g}$ entwickeln kann.

Synonym: Wechselfieber, Sumpffieber.

Englisch: malaria

\section{Ätiologie/Pathogenese}

Die Malaria wird durch Hämatosporidien der Gattung Plasmodium hervorgerufen: P. vivax, P. ovale, P. malariae, P. falciparum. Die Übertragung erfolgt durch die weibliche Anopheles-Mücke, selten durch Bluttransfusion oder transplazentar.

Mücke: Die Erreger durchlaufen im Insekt einen sexuellen Entwicklungszyklus (Sporogonie), der mit der Bildung

71 Malaria stammt aus dem Italienischen und bedeutet „schlechte Luft“. der Sporozoiten (infektiösen Sichelkeimen), die sich in der Speicheldrüse ansammeln, endet. Beim Stich infiziert die Anopheles-Mücke bei der Blutmahlzeit den Menschen.

Leber: Die Sporozoiten gelangen nun hämatogen in die Leber, in dessen Parenchymzellen eine ungeschlechtliche Vermehrung (Schizogonie) stattfindet. In den betroffenen Zellen entwickelt sich der Schizont, der wiederum in unzählige Merozoiten zerfällt. Diese präerythrozytäre oder exoerythrozytäre Phase dauert je nach Plasmodienart 5-18 Tage und bestimmt die Inkubationszeit bis zum ersten Auftreten eines Fieberanfalls (durchschnittlich 10-14 Tage bei P. ovale, P. vivax und P. falciparum; 18 Tage bis 8 Wochen bei P. malariae).

Erythrozyten: Die freigesetzten Merozoiten befallen nun via spezifischer Rezeptoren zirkulierende rote Blutkörperchen (endoerythrozytäre Phase), in welchen es erneut $\mathrm{zu}$ einer ungeschlechtlichen Vermehrung kommt. Der Zerfall der Erythrozyten, bei welchen eine neue Generation von Merozoiten freigesetzt wird, ist begleitet von hohen Fieberschüben aufgrund pyogener Stoffwechselund Abbauprodukte der Parasiten. Diese neuen Merozoiten befallen wiederum Erythrozyten, sodass dieser Zyklus fortgesetzt wird. Einige Merozoiten entwickeln sich zu Gametozyten (Gametogonie), die wieder in die Anopheles-Mücke gelangen können, sodass sich der Generationszyklus hier schließt.

\section{Klinik}

Je nach Erreger wird in eine Malaria tertiana (P. vivax, P. ovale), M. quartana (P. malariae) und M. tropica (P. falciparum) unterschieden, die auch differente klinische Verläufe zeigen. Diese entstehen 
durch die unterschiedlichen Reifungszeiten der Erreger, die sich in verschieden langen Intervallen der Fieberschübe äußern.

Der Vermehrungszyklus bei Plasmodium falciparum und vivax beträgt jeweils 48 Stunden, bei Plasmodium ovale ca. 50 Stunden sowie beim Plasmodium malariae ca. 72 Stunden. Daher bestehen bei der Infektion mit Plasmodium malariae jeweils zwei fieberfreie Tage zwischen den einzelnen Fieberschüben, wodurch das Fieber nur jeden ersten und vierten Tag auftritt, woraus sich die Bezeichnung Malaria quartana erklärt. Plasmodium vivax und Plasmodium ovale bewirken einen Fieberschub, der jeden dritten Tag zu verzeichnen ist $\rightarrow$ Malaria tertiana. Die Malaria quotidiana stellt eine Sonderform mit täglich Fieberschüben dar, deren Ursache zwei unterschiedliche, um 24 Stunden verschobene, Plasmodiengenerationen ( $\mathrm{P}$. vivax oder ovale) sind. Bei der Malaria tropica (P. falciparum), der schwersten und gefährlichsten Form sind die Fieberschübe häufig durch Überlagerung verschiedener Zyklen und Mehrfachinfektionen unregelmäßig.

Klinisch treten anfangs meist unspezifische, allgemeine Krankheitssymptome wie z.B. Kopfschmerzen, Müdigkeit, Übelkeit, Erbrechen, Durchfälle und nur leichtes Fieber auf. Nach Ablauf mehrerer Reproduktionszyklen kommt es zu Schüttelfrost, Fieberschüben mit Temperaturen bis über $40{ }^{\circ} \mathrm{C}$, Anämie, Splenomegalie (tropisches Splenomegaliesyndrom mit der Gefahr der Milzruptur). Bei der gefährlichen Malaria tropica können Komplikationen wie z.B. eine Niereninsuffizienz (Tubulusnekrosen, Hämoglobinurie $\rightarrow$ Schwarzwasserfieber), Lungenödem mit respiratorischer Insuffizienz (ARDS), Gerinnungsstörungen durch
Thrombozytämie, weitere Infekte durch Leukozytopenie, Krampfanfälle, Bewusstseinsstörungen bis hin zum Koma hinzutreten. Diese zerebralen Symptome der Malaria tropica sind durch Erythrozytensequester mit begleitender Zirkulationsstörung verursacht.

Von großer Bedeutung ist, dass durch eine mögliche Erregerpersistenz von 5 (P. vivax und P. ovalae) bis sogar 50 Jahren (P. malariae) eine erneute oder de novo Erkrankung nach diesen vielen Jahren möglich ist, sodass ein Tropenaufenthalt nicht unmittelbar vor Erkrankungsbeginn stattgefunden haben muss. Wiederholte Infektionen bewirken eine gewisse Immunität, die zu einem mehr oder weniger ausgeprägten asymptomatischen Verlauf führen kann. Eine natürliche Immunität gegen P. vivax besitzen vor allem dunkelhäutige Personen mit Duffy-negativer Blutgruppe, da der Rezeptor an der Erythrozytenmembran fehlt. Auch Hämoglobinopathien wie das Hämoglobin S (Sichelzellanämie) oder der Glukose-6-Phosphat-DehydrogenaseMangel sind mit einem gewissen Schutz assoziiert.

Die Diagnose erfolgt mittels PCR und auch mit Hilfe eines Giemsa gefärbten Blutausstrichs.

Chloroquin und Chinin sind die klassischen Therapeutika, wobei interessant ist, dass jüngst ein pflanzlicher Wirkstoff entdeckt worden ist, der Parasiten abtöten kann. Es handelt sich um die Artemisia annua (einjähriger Beifuss), die sich vor allem in den Bergregionen von Südchina und Vietnam findet und dort, durch die für diese Pflanze optimalen Wachstumsbedingungen, die höchste Konzentration des Wirkstoffs Artemisinin enthält. 


\section{Morphologie}

Die stark blutgefüllte und vergrößerte Milz enthält Retikuloendothelialzellen und Makrophagen, die reichlich so genanntes Malariapigment (Hämozoin) in ihren Zytoplasma aufweisen. Die Leber kann ebenso vergrößert sein und durch Hypertrophie und Hyperplasie der Kupfferschen Sternzellen auffallen, die Pigment, Parasiten und Zelldetritus im Zellleib aufweisen. In der Niere findet sich eine Stauungshyperämie und eine Vergrößerung mit Pigmentablagerungen. Durch das P. falciparum können neben einer Hyperämie der Hirngefäße Ringblutungen mit zentraler ischämischer Nekrose und Mikrogliareaktion (Dürck'sche Granulome) entstehen.

\section{Literatur}

Bray PG, Ward SA, O’Neill PM (2005) Quinolines and artemisinin: chemistry, biology and history. Curr Top Microbiol Immunol 295: 3-38.

Idro R, Jenkins NE, Newton CR (2005) Pathogenesis, clinical features, and neurological outcome of cerebral malaria. Lancet Neurol 4 (12): 827-40.

Roberts DJ, Casals-Pascual C, Weatherall DJ (2005) The clinical and pathophysiological features of malarial anaemia. Curr Top Microbiol Immunol 295: 137-67.

\section{V.7 Haemosiderosis pancreatis. Anaemia haemolytica secundaria}

\section{Kasuistik}

Ein 45-jähriger Buchhalter gelang wegen einer Anämie zur Aufnahme. Schon seit 4 Jahren war er wegen einer makrozytären Anämie in Behandlung. Eine Splenektomie erfuhr er 1 Jahr zuvor. Unter Cortison und B12-Medikation Besserung, dann aber blutendes Ulcus duodeni, Exitus mit den Zeichen der kardialen Insuffizienz. Der Obduktionsbefund erbrachte eine Haemosiderosis gravis universalis sowie eine periphere Pulmonalembolie mit Lungeninfarkt.

\section{Morphologische Untersuchung}

Ein tief braunes, muskelartig imponierendes, gelapptes Pankreas. Histologisch ein gering diffus fibrosiertes Pankreas mit reichlich Hämosiderophagen sowie Pigmenteinlagerungen in den Azinuszellen. Die zentroazinären Zellen und die Gangepithelien weitgehend frei von diesen.

\section{Diagnose}

Haemosiderosis pancreatis. Anaemia haemolytica secundaria. MN 6986 (Abb. V.7 $\rightarrow$ Farbbild S. 182) - Hämosiderose des Pankreas.

\section{Definition}

Bei der Haemosiderosis pancreatis handelt es sich um eine eher selten auftretende Mitbeteiligung der Bauchspeicheldrüse im Rahmen eines generalisierten Eisenüberschusses, kaum bei primärer ( = Hämochromatose; Brozediabetes), aber häufiger bei sekundärer Hämosiderose. 


\section{Geschichtliche Notizen}

Die Erkrankung des Bronzediabetes wurde von Charles Emile Troisier (18441919) erstmals 1871 beschrieben, wobei 1882 Hanot und Chauffard die assoziierte Leberzirrhose feststellten. Die Bezeichnung Hämochromatose erhielt sie von Friedrich Daniel von Recklinghausen (1833-1910) im Jahr 1889.

\section{Ätiologie/Pathogenese}

Die primäre Hämosiderose oder die Hämochromatose (Morbus von Recklinghausen-Appelbaum) ist eine autosomal rezessive Erkrankung, die durch eine gesteigerte Eisenresorption $\mathrm{zu}$ einer massiven Anhäufung von eisenhaltigem Pigment im Körper führt. Die Genveränderungen hierfür sind am kurzen Arm des Chromosom 6 lokalisiert worden, wobei 1996 Mutationen (H63D; C282Y) im hfe-Gen als molekulare Ursache identifiziert wurden. Das resultierende HFE Protein gehört zur MHC Klasse I und findet sich vor allem in den Dünndarmepithelien der tiefen Krypten im Duodenum sowie in der Leber nur an Kupfferschen Sternzellen und Endothelien. HFE wird im Fall der Mutationen vermindert an der Oberfläche exprimiert, wo es normalerweise mit dem Transferrinrezeptor interagiert. Es erfolgt auch eine verminderte Affinität zwischen Rezeptor und Transferrin, sodass das intrazelluläre Eisen akkumuliert. Die Penetranz ist aber durch weitere Faktoren bestimmt, da selbst homozygote Familien nicht immer an der Erkrankung leiden. Heterozygote weisen lediglich einen gering erhöhten Eisenspiegel im Serum und den Geweben auf. Darüber hinaus ist auch eine juvenile Hämochromatose bekannt, die um das 20. Lebensjahr auftritt, die nicht auf eine Mutation des hfe-Gens zurückgeführt werden kann, sondern deren Gendefekt auf 1q lokalisiert ist.

Eine Eisenüberladung kann jedoch auch auf andere Ursachen zurückgehen ( $=$ sekundäre Hämosiderose). Eine generalisierte Hämosiderinablagerung in phagozytierenden Zellen, aber auch in Parenchymzellen, findet sich bei systemischer Eisenüberladung und wird als (Hämo-)Siderose bezeichnet. Entstehen kann sie als Folge einer vermehrten Eisenzufuhr oder Eisenresorption, einer vermehrten Eisenfreisetzung bei Hämolyse oder einer verminderten Eisenverwertung. Aufgrund einer nutritiv-toxischen Leberschädigung sollte infolge einer Proteinsynthesehemmung und eines Apoferritinmangels durch Alkohol die sog. „Bantu“-Siderose $\mathrm{e}^{72}$ ausgelöst werden. Andere Ursachen sind wiederholte Bluttransfusionen (Transfusionssiderose) oder vermehrter Erythrozytenzerfall bei chronischen hämolytischen Anämien (generalisierte Hämosiderose). Im Gegensatz zur primären Hämochromatose steht bei diesen Sideroseformen die Hämosiderinablagerung in Zellen des RHS im Vordergrund und erst später in den Parenchymzellen. Das heißt, dass es im Gegensatz zu den primären Formen anfangs zu keinen Parenchymnekrosen kommt. Die Beteiligung vom Pankreas, endokrinen Drüsen und dem Herzen ist eher selten.

72 Bantu ist ein obsoleter Begriff für eine sehr große multiethnische Gruppe von Afrikanern mit ähnlicher gemeinsamer Sprache. Die Bantu-Siderose trat bei häufigem Genuss von eisenreichem Bier, das traditionell in Stahlfässern gebraut wurde, auf. Die Eisenverteilung ist vorwiegend in nicht parenchymatösen Zellen lokalisiert und damit ungleich dem Hämochromatose-Muster. Dennoch dürften auch genetische Veränderungen beteiligt sein. 
Außerdem kommt es zu Eisenablagerung im Gefolge spezieller Autoimmunerkrankungen wie z. B. der idiopathischen Lungenhämosiderose und dem GoodpastureSyndrom.

\section{Klinik}

Die Hämochromatose tritt bei Männern um das 40., bei Frauen um das 50. Lebensjahr auf. Folgen der Eisenüberladung sind eine Leberzirrhose, eine pluriglanduläre Insuffizienz, u.a. eine progrediente Pankreasfibrose (sog. Pankreaszirrhose) und daraus resultierend ein Diabetes mellitus (70\%), der wegen der gleichzeitig bestehenden bronzefarbenen Hauptpigmentierung (80 \%) auch als „Bronzediabetes“ bezeichnet wird. In $60 \%$ der Fälle ist ein pathologisches EKG zu verzeichnen. Aus pathogenetisch noch ungeklärter Ursache tritt bei einem Teil der Patienten vor allem im Fingerund Handbereich eine Arthropathie mit Chondrokalzinose (25\%) auf. Außerdem findet sich als regelmäßiger hämatologischer Befund eine Plasmazellsiderose. Dabei wird Eisen grobschollig intralysosomal in der Nähe des Kernhofes abgelagert.

Da im Rahmen der pluriglandulären Insuffizienz auch andere endokrine Funktionen mit beteiligt sind, kann es weiters $\mathrm{zu}$ einem Hypopituitarismus und einer Schädigung der gonadotropinproduzierenden Zellen kommen.

Zur diagnostischen Unterscheidung einer primären Siderose (= Hämochromatose) und einer sekundären Siderose ist eine Magenschleimhautbiopsie wegweisend: Bei der Hämochromatose speichern die Epithelien der Korpusdrüsen Hämosiderin, bei den sekundären Siderosen jedoch praktisch nie.

Die Therapie erfolgt durch Entleerung der Eisenspeicher durch Aderlass (wöchentlich $500 \mathrm{ml}$ über 2 Jahre). Die Prognose hängt von der Frühdiagnose und rechtzeitigen Behandlung ab. Das Risiko an einem hepatozellulären oder cholangiozellulären Karzinom zu erkranken beträgt bei Vorliegen einer Leberzirrhose $14 \%$ ! Das Risiko wird auch durch die Aderlässe nicht verringert.

\section{Morphologie}

Das Pankreas weist eine derbe Konsistenz und eine rostbraune Farbe auf. Histologisch finden sich Hämosiderinablagerungen in den Parenchymzellen sowie Bindegewebszellen. Hämosiderin ist ein gelbbraunes, grobgranuläres Pigment, das aus Ferritin-Aggregaten besteht. Das Pankreas zeigt überdies eine Fibrose mit Atrophie des inkretorischen und exkretorischen Parenchyms.

In der Leber prägen Parenchymnekrosen mit Fibrose und schließlich ein zirrhotischer Umbau mit charakteristischer rostbrauner Verfärbung das Bild der Pigmentzirrhose. Im subepidermalen Bindegewebe sind ebenso Eisenablagerungen, sowie eine Melaninanreicherung in der Epidermis feststellbar, wobei letztere durch eine Störung der Tyrosinaseinhibition im Rahmen der Bindung von Eisen an Glutathion verursacht wird. Weiters ist eine vermehrte Speicherung im RES der Milz, der Lymphknoten und des Knochenmarks mit anschließender Fibrosierung zu verzeichnen. 
Literatur

Bosserhoff AK, Hellerbrand C, Buettner R (2001) Klinische und genetische Aspekte der hereditären Hämochromatose. Pathologe 22/3: 191-196.

Hanot VC, Chauffard AME (1882) Cirrhose hypertrophique pigmentaire dans le diabète sucré. Revue de médecine, Paris 2: 385-403.

Recklinghausen FD (1889) Über Hämochromatose. Berliner Klin Wochenschr 26: 925.

Troisier CE (1871) Diabète sucré. Bull Société anatomique de Paris 16: 231.

\section{V.8 Adenoma pancreatis \\ (Adenoma insulare)}

\section{Kasuistik}

MN 8278: Eine 46-jährige Frau.

\section{Morphologische Untersuchung}

Ein $3: 3,5: 2,5 \mathrm{~cm}$ großer zirkumskripter bräunlicher Tumor im Caput pancreatis mit teils feinwabiger bis homogener Schnittfläche. Der Ductus pancreaticus gering eingeengt. Das umliegende Pankreas grauweißlich, regulär gelappt ohne weitere makromorphologische Auffälligkeiten.

Histologisch ein Tumorgewebe mit schmalen Trabekeln sowie mehrfach pseudotubulären Strukturen. Die relativ großen Tumorzellen zeigen weitgehend monomorphe und überwiegend hypochromatische Kerne. Fokal die Kerne auch chromatindicht und klein. Das reichlich vorliegende Zytoplasma deutlich eosinophil. Mitosen nur ganz vereinzelt fassbar. Der Tumor auf weiten Strecken auch pseudozystisch aufgelockert, das wenig vorhandene, teils trabekelförmige Stroma aus hyalinsiertem, zellarmen Bindegewebe bestehend. Der Tumor wird allseits von einer mittelbreiten Bindegewebskapsel umgeben. Gefäß- oder Kapselinfiltrationen nicht feststellbar.

Immunhistochemisch eine zarte Positivität für Chromogranin $\mathrm{A}$ und mäßige Expression von Insulin. Der MIB-Index unter $1 \%$.

\section{Diagnose und Kommentar}

Ursprüngliche Diagnose: Adenoma insulare MN 8278 (Abb. V.8).

Moderne Diagnose: Hoch differenzierter, neuroendokriner Tumor des Pankreas 
mit Insulin-Expression und niedrig maligner Potenz.

Von Insulinom spricht man heute nur, wenn der Tumor funktionell aktiv ist und somit klinisch zeichnet.

Entsprechend der Klassifikation und der Dignitätskriterien (Klöppel, 2003) spricht lediglich die Größe gegen ein rein benignes Verhalten. Für einen niedrig malignen Prozess ist die Größe sicherlich nicht ausreichend. Den Tumor mit fraglicher Dignität zu bezeichnen ist vielleicht zu wenig spezifisch und diffus. Daher hat der Autor die Formulierung „niedrig maligner Potenz“ gewählt, um auszudücken, dass der Tumor prinzipiell gutartig ist, aber dennoch (wegen der Größe) bereits eines der Malignitätskriterien aufweist, aber die Wahrscheinlichkeit für malignes, wenn auch niedrig malignes Verhalten sehr gering ist.

\section{Definition}

Ein von den $\beta$-Zellen ausgehender meist benigner Tumor, der bei funktioneller Aktivität zu einem Hypoglykämie-Syndrom führt.

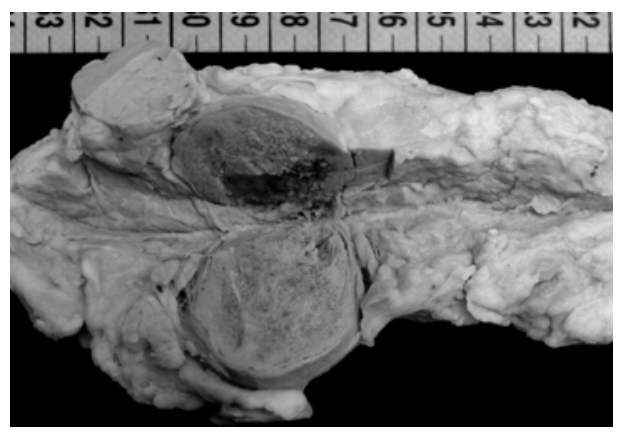

Abb. V.8. MN 8278. Adenoma insulare pancreatis

\section{Klinik}

Die autonome Insulinproduktion führt $\mathrm{zu}$ einem Hypoglykämiesyndrom mit Whipple ${ }^{73}$-Trias: Hypoglykämie (< $50 \mathrm{mg} \%$ ), Kombination mit suspekten Symptomen, Besserung dieser durch Einnahme von Kohlenhydraten.

Klassische Symptome: Whipple Trias, Schwindelanfälle mit Kollapsneigung, Gewichtszunahme.

Adrenerge Symptome: Heißhunger, Tremor, Schweißausbrüche, Kopfschmerzen, Übelkeit, Unruhe/Nervosität, Tachykardie.

Neuroglycopenische Symptome: Bewusstseinsstörungen bis -verlust, Verwirrtheit, Konzentrationsstörungen, Krampfanfälle, Müdigkeit, Doppelbilder/Sehstörungen, Paresen.

Die überwiegende Mehrheit der Insulinome sind benigne, dennoch tritt selten eine maligne Variante auf. In 5 \% der Fälle treten Insulinome multipel auf, insbesondere wenn ein Multiples Endokrines Neoplasma (MEN)-1 Syndrom vorliegt.

\section{V.8.6 Morphologie}

Makroskopisch sind die Tumoren gut begrenzt, rund und $1-5 \mathrm{~cm}$ groß. Histologisch sind diese monomorph mit solidem, trabekulärem, azinären und/oder pseudoglandulären Wachstumsmuster.

73 Allen Oldfather Whipple (1881-1963), amerik. Chirurg. Nach ihm und dem dt. Walter Kausch (1867-1928) wurde die radikale Duodenopankreatektomie benannt. Kausch führte die Operation allerdings bereits 25 jahre vor Whipple im Jahr 1909 durch, wobei der Patient von Kausch auch 1 Monat länger als der von Whipple überlebte - 9 Monate. 


\section{Literatur}

Grant CS (2005) Insulinoma. Best Pract Res Clin Gastroenterol 19 (5): 783-98.
Klöppel G (2003) Tumoren des endokrinen Pankreas. Der Pathologe 24: 265-271. Whipple AO, Frantz VK (1935) Adenoma of islet cells with hyperinsulinism: a review. Ann Surg 101: 299-1335. 